\title{
Class of Case
}

National Cancer Institute

\section{Source}

National Cancer Institute. Class of Case. NCI Thesaurus. Code C160892.

A designation assigned to a group of cases within a study or trial. 